\title{
An evaluation of people's knowledge of adult vaccination information level and attitudes during the pandemic Era
}

\author{
Neşe Kıskaç (D), Muharrem Kiskaç²* (D), Abdüsselam Şekerci² (D), Mehmet Zorlu²
}

\section{SUMMARY}

OBJECTIVE: This research was carried out to evaluate people's knowledge of adult vaccination and their attitude and to observe the effect of the pandemic era on this situation.

METHODS: A total of 1,425 people (18-80 years old) were included in this study. The types of questions like the province where they live, age, gender, occupation, education status, and the presence of chronic diseases, as well as knowing which vaccines are used in adult vaccination, which of these vaccines they had in the last 10 years, which ones they plan to have this year, and whether COVID-19 pandemic changed their perspective on adult vaccinations or not were asked to people.

RESULTS: In the last 10 years, while participants stated that they had the highest rate of tetanus vaccine with $29.8 \%$, hepatitis B vaccine with $23.1 \%$, influenza vaccine with $22.7 \%$, human papillomavirus vaccine with $1.3 \%$, and zoster vaccine with $0.3 \%$ were the lowest levels of vaccines.

CONCLUSIONS: As a result, it seems that we are far from the goals set by the health authorities for adult vaccination. We observed that the COVID-19 pandemic raised awareness toward pneumococcus and influenza vaccines and interest toward adult vaccinations and at the same time changed the thoughts against adult vaccinations.

KEYWORDS: Vaccination. Pandemic. Pneumococcal vaccines. Influenza vaccines.

\section{INTRODUCTION}

The incidence of vaccine-preventable diseases has been reduced by more than $99 \%$ after vaccination, and some of them (smallpox) have been completely eradicated ${ }^{1}$. With an effective vaccination, it is possible to reduce the number of referrals to healthcare institutions and hospitalizations, as well as by using less antibiotics and reducing infections with resistant microorganisms. Thus, a serious reduction in mortality and morbidity can be achieved. The success achieved in childhood vaccination in our country and in the world is not yet at the desired level for adult vaccination; it is still reported as a neglected issue in many countries; and solutions are being sought to increase application rates ${ }^{2,3}$.

We observed that the interest and demand for adult vaccination have increased after the effects of the COVID-19-induced pandemic on the world. The increasing number of cases and deaths, especially with the pandemic, causes fear and anxiety as well as confusion about adult vaccination. In the literature review, it is seen that there are limited studies on this subject.

This research was carried out to evaluate people's knowledge of adult vaccination and their attitude and to observe the effect of the pandemic era on this situation.

\footnotetext{
'University Institute of Health Sciences, Istanbul Provincial Health Directorate, Hospital Services Department - Istanbul, Turkey.

${ }^{2}$ Bezmialem Vakif University Faculty of Medicine Hospital, Department of Internal Medicine - Istanbul, Turkey.

*Corresponding author: muharremkiskac@gmail.com

Conflicts of interest: the authors declare there are no conflicts of interest. Funding: none.

Received on July 11, 2021. Accepted on August 10, 2021.
} 


\section{METHODS}

During the pandemic period, it was planned to conduct a descriptive study to evaluate the adult vaccination knowledge and attitudes; approval was obtained from the Ethics Committee; and patient information were collected. This research was conducted in the form of an online questionnaire, and 1,425 people aged 18 years and older were included in this study.

Participants who voluntarily accepted to participate in this research were informed about the research and their rights, and their "informed consent" was obtained before the research.

The online survey for the participants consisted of 21 questions; in addition to their provincial, age, gender, occupation, education status, presence of chronic disease, marital status, presence of children, which vaccines were used in adult vaccination that they know (e.g., pneumococcus, influenza, tetanus, meningococcus, hepatitis A, hepatitis B, zoster, human papillomavirus (HPV) vaccine, etc.) or which of these vaccines they had in the last 10 years, which ones they plan to have this year, whether COVID-19 pandemic has changed their view on adult vaccines, the protectiveness of the pneumococcal and influenza vaccine, its usage periods, risk groups, and its effects on COVID-19, and their perspectives on coronavirus vaccines in production were asked.

IBM SPSS statistics version 22.0 program was used for statistical analysis in this study. While evaluating the data of this study, besides descriptive statistical methods (i.e., mean, standard deviation, and frequency), the chi-square test was used to evaluate the relationship between variables, the Student's $t$-test for the comparison of normally distributed data, and the Mann-Whitney $U$ test for comparing non-normally distributed data. Results were evaluated at $95 \%$ confidence interval and significance level of $\mathrm{p}<0.05$.

\section{RESULTS}

A total of 1,425 people were included in this study, including $850(59.6 \%)$ women and $575(40.4 \%)$ men. The mean age of the participants was $37.4 \pm 10.78$ years, while the men were $37.73 \pm 10.64$ years and the women were $37.19 \pm 10.88$ years, and there was no statistically significant difference in age between women and men ( $\mathrm{p}>0.05)$.

Among 286 out of 1,425 people participating in this study, they had one or more chronic diseases. While the mean age of this group was $44.40 \pm 11.58$ years, the mean age of 1,139 people without chronic disease was $36.65 \pm 9.82$ years. There was a statistically significant difference in age between the groups $(\mathrm{p}<0.05)$.

In adult vaccination, the highest awareness rate was the tetanus vaccine with $96.6 \%$, while the lowest awareness rate was the zoster vaccine with $27.6 \%$ (Table 1 ). While there was no difference in awareness between the genders in the zoster vaccine ( $p>0.05)$, the awareness of all other vaccines was statistically significantly higher in women. No significant difference was found in comparing the individuals' awareness of vaccine with and without chronic diseases.

When we compared the patients above the age of 35 years with those below the age of 35 years, the HPV vaccine was significantly higher in those who aged above 35 years, but there was no statistically significant difference in terms of other vaccines.

Vaccines received by the participants in the last 10 years are shown in Table 2 . The vaccines that the participants plan to have this year are shown in Table 3 .

Table 1. Awareness levels of vaccines used in adult vaccination.

\begin{tabular}{l|c}
\hline Adult vaccines & $(\%)$ \\
\hline Tetanus vaccine & 96.3 \\
\hline Hepatitis B vaccine & 91.9 \\
\hline Influenza vaccine & 91.6 \\
\hline Pneumococcal vaccine & 86.7 \\
\hline Hepatitis A vaccine & 74.7 \\
\hline Meningococcus vaccine & 73 \\
\hline Human papilloma virus vaccine & 50.4 \\
\hline Zoster vaccine & 27.6 \\
\hline
\end{tabular}

Table 2. Levels of adult vaccines that people have had in the last 10 years.

\begin{tabular}{l|c}
\hline Adult vaccines & $(\%)$ \\
\hline Tetanus vaccine & 29.8 \\
\hline Hepatitis B vaccine & 23.1 \\
\hline Influenza vaccine & 22.7 \\
\hline Pneumococcal vaccine & 8.6 \\
\hline Hepatitis A vaccine & 6.5 \\
\hline Meningococcus vaccine & 2 \\
\hline Human papilloma virus vaccine & 1.3 \\
\hline Zoster vaccine & 0.3 \\
\hline
\end{tabular}

Table 3. Levels of adult vaccines people plan to have this year.

\begin{tabular}{l|c}
\hline Adult vaccines & $(\%)$ \\
\hline Influenza vaccine & 30.3 \\
\hline Pneumococcal vaccine & 17.8 \\
\hline Hepatitis B vaccine & 5.5 \\
\hline Tetanus vaccine & 5.1 \\
\hline Human papilloma virus vaccine & 3.4 \\
\hline Hepatitis A vaccine & 2.2 \\
\hline Meningococcus vaccine & 0.6 \\
\hline Zoster vaccine & 0 \\
\hline
\end{tabular}


To the question we asked the participants "Have coronavirus pandemic changed your perspective on adult vaccinations?" $886(62.2 \%)$ participants answered as "No, it hasn't," while $539(37.8 \%)$ participants answered as "I'm thinking of having one or more than one of these vaccines that I have not thought about having before the coronavirus pandemic for myself or one of my family members."

When we asked the question about the effects of pneumococcal vaccine on pneumonia and COVID-19 infection, 930 (65.2\%) participants said, "This vaccine does not protect against either coronavirus or pneumonia, it only protects from pneumococcal pneumonia," $380(26.6 \%)$ people said, "This vaccine is not protective against coronavirus, but I think it protects from pneumonia caused by coronavirus," and 115 $(8.1 \%)$ people responded as "I think this vaccine protects against coronavirus."

To the question we asked about the effects of influenza vaccine on influenza and COVID-19 infection, 1,294 (90.8\%) participants answered as "I do not think this vaccine protects against coronavirus, I think it only prevents influenza infection caused by influenza virus," while 131 (9.2\%) people answered as I think this vaccine protects against coronavirus" in the form.

Participants answered the question "To whom should the pneumococcal and influenza vaccine be given first?" The most common answer was "people over 65 , those with chronic diseases and healthcare workers."

To the question we asked about coronavirus vaccines in production, 552 (38.7\%) people answered as "I do not think that a fully effective vaccine can be produced against coronavirus," $672(47.2 \%)$ people answered as "I think the vaccine to be produced against coronavirus will be partially or shortterm effective," and 201 (14.1\%) people answered as "I think the vaccine to be produced against coronavirus will be completely effective."

Finally, to the question "If the coronavirus vaccine is produced, do you plan to have it?" 562 (39.4\%) people answered as "If I see that there is no serious side effect in those who had the vaccine for a while after the vaccine is produced, I will have it done later," 270 (18.9\%) people answered as "I consult my doctor and decide according to their suggestions," 159 (11.2\%) people said, "Yes, I'll immediately have it." In addition, 278 (19.5\%) people answered "I'm undecided," and 156 (10.9\%) people answered as "I will definitely not have it."

\section{DISCUSSION}

Despite the increasing efforts in recent years, due to reasons such as people have doubts about the efficacy and side effects of vaccines, misleading news, anti-vaccination movements, insufficiency of national health policies on adult immunization, and the economic burden the vaccination brings, the literature reveals that only $10-20 \%$ of the targeted adult vaccination groups can be vaccinated ${ }^{4}$. Due to the COVID-19 pandemic, we observed that the interest and demand for adult vaccines, especially pneumococcal and influenza vaccines, have increased in 2020. When we considered the findings of our study, in the last 10 years, the highest rates of vaccines received by the participants were tetanus vaccine with $29.8 \%$, hepatitis $B$ vaccine with $23.1 \%$, and influenza vaccine with $22.7 \%$. In the study by Kanitz et al., they found that the most commonly used vaccine in European countries is the influenza vaccine, followed by the tetanus vaccine 5 . In the study performed by Özışı et al. in our country with 522 patients, while it was found that the most commonly used vaccine was the tetanus vaccine, the most commonly used vaccine in patients with chronic lung disease, cardiovascular diseases, and diabetes was found to be influenza vaccine $^{6}$. The results found in the study by Özışı et al. ${ }^{6}$ are in line with the results of our study. The reason for this is that the tetanus vaccine is routinely administered to prevent neonatal tetanus during pregnancy before compulsory military service in our country, and it is thought that tetanus vaccine is the most known and administered vaccine due to the administration of tetanus vaccine after trauma and accidents. Unlike the study conducted by Özışık et al. ${ }^{6}$, in our study, the tetanus vaccine was the most commonly used vaccine in patients with chronic diseases within 10 years, followed by the influenza vaccine ${ }^{6}$. The reason for this difference is thought to be due to the fact that chronic diseases were wider in our study, the number of patients was higher, the 10-year process was questioned, and the patient profiles were different.

In our study, there was no significant difference between men and women in terms of adult vaccines they had in the last 10 years, except for HPV (more common in women) and zoster (more common in men) vaccines. However, the awareness rate in all adult vaccines, except for the zoster vaccine, was statistically significantly higher in women. This reveals that awareness is higher about adult vaccination in women, although it is not yet fully implemented in practice.

In our study, HPV and zoster vaccines were the least vaccines that individuals had in the last 10 years. The study in which Yuruyen et al. evaluated the awareness of physicians in adult vaccination performed on 200 physicians from different branches in our country, the vaccines recommended by the physicians to patients were the lowest with $1 \%$ while the rate of recommending the HPV vaccine was found to be $4.5 \%{ }^{7}$. Since it is not recommended a lot by physicians and its payment is not covered by social security institutions, it is expected that these vaccines will have the lowest rate in our country. 
Again, in the same study, the three vaccines frequently recommended by physicians with a rate of $22 \%$ are influenza, hepatitis $B$, and pneumococcal vaccines ${ }^{7}$. The low rates of these recommendations can be considered as one of the main reasons for not achieving the goals in adult vaccination. In the study performed by Özışı et al. ${ }^{6}$ on 155 patients, it was found that 93.5\% of the patients had the indication for influenza and pneumococcal vaccines, but only $17.2 \%$ of these patients had the pneumococcal vaccine and $29.7 \%$ had the influenza vaccine. When we asked the patients who were vaccinated, they answered that they had the highest rate of vaccinations because their physicians recommended it ${ }^{8}$. Considering the studies conducted, it is seen that the rate of vaccination is affected by the doctor's recommendation. Similarly, there is evidence that the majority of patients will be vaccinated if recommended by healthcare providers ${ }^{8-10}$.

When we asked the participants in our study "Have coronavirus pandemic changed your perspective to vaccines?" 539 $(37.8 \%)$ people replied as "I'm thinking of having one or more than one of these vaccines that I have not thought about having before the coronavirus pandemic for myself or one of my family members," and again when we asked these individuals which vaccines they plan to have in this year, it was stated by them that they will have influenza vaccine, especially with the highest rates of 30.3 and $17.8 \%$ pneumococcal vaccine later. These rates were 1.5 times for influenza vaccination and two times for pneumococcal vaccine more than the rates that patients stated that they had received these vaccines in the last 10 years. These results show that the pandemic has led to an increased interest in adult vaccines, especially toward influenza and pneumococcal vaccines.

In our study, when we asked the patients about the effects of pneumococcal and influenza vaccines on coronavirus infection, pneumonia, and influenza, 380 (26.6\%) people said, "This vaccine is not protective against coronavirus, but I think it protects from pneumonia caused by coronavirus" for the pneumococcal vaccine and $115(8.1 \%)$ people answered as "I think this vaccine protects against coronavirus," while 131 (9.2\%) people answered as "I think this vaccine protects against coronavirus" for the influenza vaccine. These responses can be considered as the main reason for the serious increase in interest in these vaccines, as there is a perception forming in the society that these vaccines are directly effective against coronavirus.

In our study, to the question we asked about the coronavirus vaccines that are in the production phase, $672(47.2 \%)$ people with the highest rate answered as "I think the vaccine to be produced against coronavirus will be partially or shortterm effective." This shows that although the participants are hopeful against the coronavirus vaccine, the expectation that a fully effective vaccine will be produced is less.

Finally, to the question "If a coronavirus vaccine is produced do you plan to have it?" only 159 (11.2\%) people have answered as "Yes, I definitely do." This situation shows that the participants harbor doubts about the COVID-19 vaccines in the production phase.

When we evaluated the limitations of our study, although there was participation from all around Turkey, the majority of the contributions from urban areas, rural areas may have led to an inadequate reflection of the data.

\section{CONCLUSIONS}

As a result, it seems that we are far from the goals set by the health authorities for adult vaccination. We observed that the COVID19 pandemic raised awareness toward pneumococcus and influenza vaccines and interest toward adult vaccinations, and at the same time it changed the thoughts against adult vaccinations. To achieve the desired goals of adult vaccination, we think that national and international organizations should work in cooperation with individuals, society, and authority-based regulations.

\section{AUTHORS" CONTRIBUTIONS}

MZ: Conceptualization, Data curation, Writing - original draft. MK: Conceptualization, Data curation, Supervision, Writing - review \& editing. NK: Writing - original draft. AS: Investigation, Methodology, Data curation.

\section{REFERENCES}

1. Akkaya N, Camcioğlu Y, Gür E, Öztürk R. Çocuk ve Erişkinlerde Aşılama. Istanbul: IU Cerrahpasa Tıp Fak Surekli Tip Egitimi Etkinlikleri; 2010. p.71.

2. Chakravarthi PS, Ganta A, Kattimani VS, Tiwari RV. Adult immunization-need of the hour. J Int Soc Prev Community Dent. 2016;6(4):272-7. https://doi.org/10.4103/2231-0762.186797

3. Kimmel SR, Burns IT, Wolfe RM, Zimmerman RK. Addressing immunization barriers, benefits, and risks. J Fam Pract. 2007;56(2 Suppl Vaccines):S61-9. PMID: 17270112
4. Toprak D, Köksal I, Sargin M, Akan H. Adult vaccination, problems in practice and solution proposals, role of family physicians in adult vaccination. Türk Aile Hek Derg. 2018;22(3):166-74. https://doi.org/10.15511/tahd.18.00366

5. Kanitz EE, Wu LA, Giambi C, Strikas RA, Levy-Bruhl D, Stefanoff $P$, et al. Variation in adult vaccination policies across Europe: an overview from Venice network on vaccine recommendations, funding and coverage. Vaccine. 2012;30(35):5222-8. https:// doi.org/10.1016/j.vaccine.2012.06.012 
6. Ozisik L, Basaran NC, Oz SG, Guven GS, Tanriover MD. Perceptions and attitudes of patients about adult vaccination and their vaccination status: still a long way to go? Med Sci Monit. 2017;23:3178-84. https://doi.org/10.12659/msm.901856

7. Yürüyen G, Toprak DI, Kutlu Y. Adult vaccination awareness in physicians. Bosphorus Med J. 2019;6(3):89-93. https://doi. org/10.14744/bmj.2019.25733

8. Özişık L, Yekedüz E, Tanrı̈ver MD, Helvaci Ö, Başaran NÇ, Unal S. Risk Altındaki Erişkinlerin Pnömokok ve İnfluenza Aşılanma Oranları ve Aşıya Karşı Tutumları. Flora. 2016 [cited on May
5, 2021];21(1):15-20. Available from: https://app.trdizin.gov. tr/publication/paper/detail/TWpRNE1UUXhNUT09

9. Johnson DR, Nichol KL, Lipczynski K. Barriers to adult immunization. Am J Med. 2008;121(7 Suppl 2):S28-35. https://doi.org/10.1016/j.amjmed.2008.05.005

10. Wheelock A, Thomson A, Sevdalis N. Social and psychological factors underlying adult vaccination behavior: lessons from seasonal influenza vaccination in the US and the UK. Expert Rev Vaccines. 2013;12(8):893-901. https://doi.org/10.1586/ 14760584.2013 .814841 